\title{
Modeling the pressure inactivation dynamics of Escherichia coli
}

K. Yamamoto, M. Matsubara,

S. Kawasaki, M.L. Bari and S. Kawamoto

\author{
National Food Research Institute, Tsukuba, Ibaraki, Japan
}

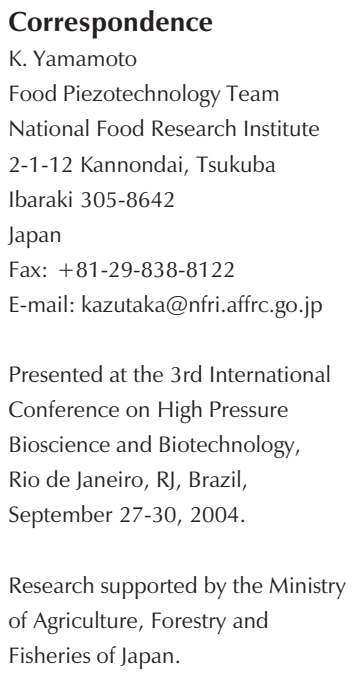

\begin{abstract}
Escherichia coli, as a model microorganism, was treated in phosphate-buffered saline under high hydrostatic pressure between 100 and $300 \mathrm{MPa}$, and the inactivation dynamics was investigated from the viewpoint of predictive microbiology. Inactivation data were curve fitted by typical predictive models: logistic, Gompertz and Weibull functions. Weibull function described the inactivation curve the best. Two parameters of Weibull function were calculated for each holding pressure and their dependence on holding pressure was obtained by interpolation. With the interpolated parameters, inactivation curves were simulated and compared with the experimental data sets.
\end{abstract}

Key words

- Predictive microbiology

- Pressure

- Escherichia coli

- Inactivation

- Model

\section{Introduction}

There has been a great increase in consumer demand for fresh and minimally processed food products. Non-thermal processing of the novel food products has received great attention. High (hydrostatic) pressure processing is one of the non-thermal methods used to produce higher quality foods more economically (1). High-pressure treatment of food, which can inactivate microorganisms and detrimental enzymes with retention of taste, texture, color, or nutrients, has an advantage over heat treatment which may cause deterioration of beneficial food components. Most of the common bacterial food-borne pathogens such as Campylobacter jejuni (2), Salmonella (3,4), Vibrio spp. (5), Escherichia coli O157:H7 (4,6), Yersinia enterocolitica $(4,7)$, and Listeria monocytogenes $(4-6,8)$ have been investigated in a variety of food systems and buffers, at various temperatures and for various pressureholding times. However, much fewer studies have been conducted on high-pressure inactivation of microorganisms, especially foodborne pathogens, than on thermal inactivation, especially in terms of predictive microbiology. Predictive microbiology represents quantitative microbial ecology of food by describing microbial responses to the food environments by mathematical models $(9,10)$.

Regarding heat sterilization, international databases $(11,12)$ and predictive modeling programs $(11,13)$ have been developed, while the study of predictive microbiology for pressure treatment of microorganisms has just started. Therefore, it is necessary to accumulate as many data as possible and to develop suitable predictive models in order to disseminate high pressure processing further.

Predictive models for pathogenic micro- 
organisms are considered to be effective tools for microbial risk assessment (14) according to the Codex Alimentarius Commission, which is the working body of the Joint FAO/WHO Food Standards Program. Furthermore, predictive microbiology will make it possible to predict inactivation dynamics of pathogenic microorganisms and to optimize high-pressure food processing.

In the present study, high-pressure inactivation of E. coli was carried out, and the data were applied to predictive microbiology.

\section{Material and Methods}

E. coli ATCC25922 was used as a nonpathogenic model microbe. A frozen stock culture was thawed and a loopful of the culture was inoculated into trypticase soy broth (TSB) and incubated at $37^{\circ} \mathrm{C}$ for $18 \mathrm{~h}$. The overnight-grown cultures in logarithmic phase $(1 \mathrm{ml})$ were transferred to $5 \mathrm{ml}$ of TSB medium and incubated at $37^{\circ} \mathrm{C}$ for $1 \mathrm{~h}$. After incubation, $1 \mathrm{ml}$ of the culture was further inoculated into $5 \mathrm{ml}$ of the medium and incubated at $37^{\circ} \mathrm{C}$ until the optical density of the culture was between 0.9 and 1.1. This process required approximately $1.5 \mathrm{~h}$ after the final inoculation. The cell suspension was then diluted 10 -fold with $0.3 \mathrm{mM}$ phosphate-buffered saline, pH 6.8. Ten milliliters of the diluted samples was then transferred to individual retort plastic bags $(5.0 \mathrm{x}$ $8.0 \mathrm{~cm}$ ) and heat-sealed. Individual plastic bags were double bagged $(7.0 \times 10.0 \mathrm{~cm})$ and sealed to prevent leakage of the contents during high-pressure treatment. All the individually sealed plastic bags were kept at $25^{\circ} \mathrm{C}$ for $60 \mathrm{~min}$ before pressurization. The sealed samples were then treated at $25^{\circ} \mathrm{C}$ with pressures between 100 and $300 \mathrm{MPa}$ using the high-pressure equipment HYPREX R7K3-15-15 (Yamamoto Suiatsu Kogyosho Co. Ltd., Tokyo, Japan). Immediately after high-pressure treatment, packages containing inoculated samples were serially diluted in phosphate-buffered saline, and diluted and undiluted samples were plated onto Standard Method Agar (Nissui Co. Ltd., Tokyo, Japan). The plates were then incubated at $37^{\circ} \mathrm{C}$ for $18 \mathrm{~h}$ before being counted. To enhance recovery the incubation period was extended to $48 \mathrm{~h}$, but the colony forming unit (CFU) of active bacterial cells did not change significantly.

The data were fitted to three functions by using the statistical software SAS. Logistic (Equation 1) and Gompertz (Equation 3) functions, which are often used to predict microbial growth $(9,10)$, were slightly modified and used so that CFU would be equal to zero when the pressure treatment time was zero (Equations 2 and 4), and the Weibull function (Equation 5) was used with no change. In the following equations, $C F U$, $N_{0}, N_{\infty}$, and $t$ are colony forming unit, initial cell number, final cell number, and time, respectively. $B$ (apparent inactivation rate), $M$ (lag time), $n$, and $b$ are empirical parameters.

Logistic function:

$$
\begin{array}{r}
\log C F U=\log N_{0}-\left(\log N_{0}-\log N_{\infty}\right) \frac{1}{1+\exp \{B(t-M)\}} \\
\text { (Eq. 1) }
\end{array}
$$

Modified logistic function:

$\log C F U=\log N_{0}-\left(\log N_{0}-\log N_{\infty}\right) \frac{1-\exp B t}{1+\exp B t}$

(Eq. 2)

Gompertz function:

$\log C F U=\log N_{0}-\left(\log N_{0}-\log N_{\infty}\right) \exp \{-\exp B(t-M)\}$

(Eq. 3)

Modified Gompertz function:

$\log C F U=\log N_{0}-\left(\log N_{0}-\log N_{\infty}\right)\{1-\exp (1-\exp B t)\}$

(Eq. 4) 
Weibull function:

$$
\log C F U=\log N_{0}-\frac{1}{2.303}\left(\frac{t}{b}\right)^{n}
$$

\section{Results}

Logarithm of CFU was plotted as a function of holding time, and experimental inactivation data plots are shown in Figure 1 for specific holding pressures: 100, 150, 170, 200, 250, and $300 \mathrm{MPa}$. In Figure 1, the log value (0.9) was taken as "CFU $=0$ " for convenience. Most of the data sets did not follow first order kinetics and were concave upward. No E. coli were detected when the material was treated at $250 \mathrm{MPa}$ for $25 \mathrm{~min}$ or at $300 \mathrm{MPa}$ for $5 \mathrm{~min}$. Each data set for each holding pressure was applied to curve fitting by using modified logistic (Equation 2), modified Gompertz (Equation 4), and Weibull (Equation 5) functions. As indicated by solid (modified logistic function) and dashed (modified Gompertz) lines, the inactivation curves fitted by using both func- tions dropped faster than the experimental CFUs and predicted faster achievement of final counts than experimental evaluations. From the viewpoint of food safety, this earlier estimation of bacterial inactivation is inappropriate, since one may overestimate the efficiency of high-pressure inactivation of a target food-borne pathogen. On the other hand, the curves fitted by Weibull function (double-dotted dashed line) approximated the data sets the best, among the three predictive models. Thus, we selected the Weibull function and further investigated it in terms of pressure dependence of the parameters.

As described above, curve fitting was carried out for 6 holding pressures, and 6 sets of Weibull function parameters $(b$ and $n$ ) were obtained. Each fitting parameter was plotted against each holding pressure and curve fitted by using polynomial functions (Figure 2). Each data set of fitting parameters was fitted well to a polynomial function (Equation 6) and an exponential function containing a reciprocal polynomial func-
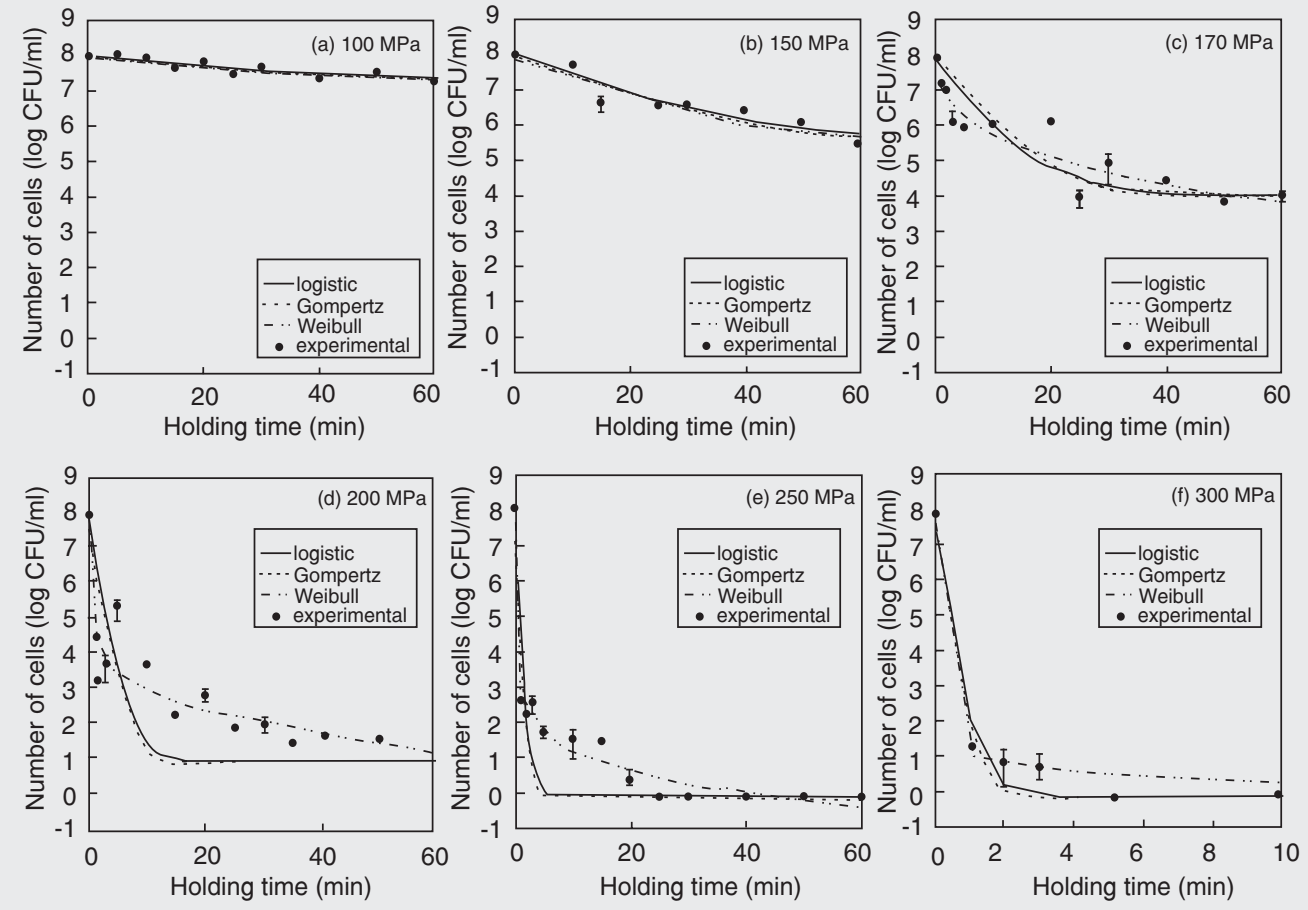

Figure 1. Curve fitting of experimental data sets of Escherichia coli inactivation by modified logistic, modified Gompertz, and Weibull functions. Holding pressure: a) $100 \mathrm{MPa}$; b) $150 \mathrm{MPa}$; c) $170 \mathrm{MPa}$; d) $200 \mathrm{MPa}$; e) 250 $\mathrm{MPa}$;) $300 \mathrm{MPa}$. 
tion (Equation 7). The degree of the polynomial functions was determined by increasing the degree one by one until the fitting curves did not change significantly.

$b=\alpha_{b}+\beta_{b} p+\chi_{b} p^{2}+\delta_{b} p^{3}+\varepsilon_{b} p^{4}+\phi_{b} p^{5}$

where $p$ represents holding pressure $[\mathrm{MPa}]$ and $\alpha_{b}, \beta_{b}, \chi_{b}, \delta_{b}, \varepsilon_{b}$, and $\phi_{b}$ are fitting parameters.

$$
n=\frac{n_{100 \mathrm{MPa}}}{\left(\frac{n_{100 \mathrm{MPa}}}{n_{300 \mathrm{MPa}}}-1\right) \exp \left(\frac{\alpha_{n}}{p}+\frac{\beta_{n}}{p^{2}}+\frac{\chi_{n}}{p^{3}}+\frac{\delta_{n}}{p^{4}}+\frac{\varepsilon_{n}}{p^{5}}+\frac{\phi_{n}}{p^{6}}\right)}
$$

(Eq. 7)
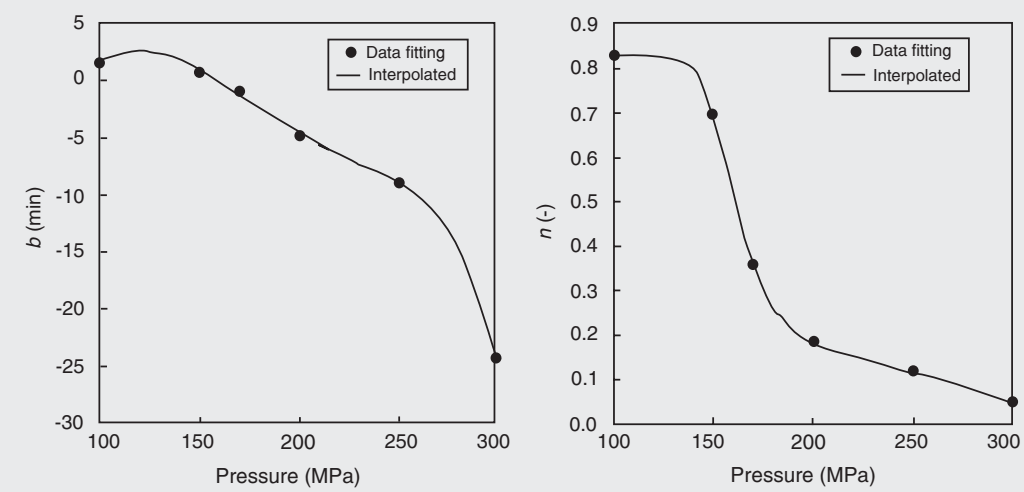

Figure 2. Dependence of Weibull function parameters $b$ (left) and $n$ (right) on holding pressure. Parameters $b$ and $n$ were interpolated using a 6th polynomial function and an exponential function of a 6th reciprocal polynomial function, respectively.

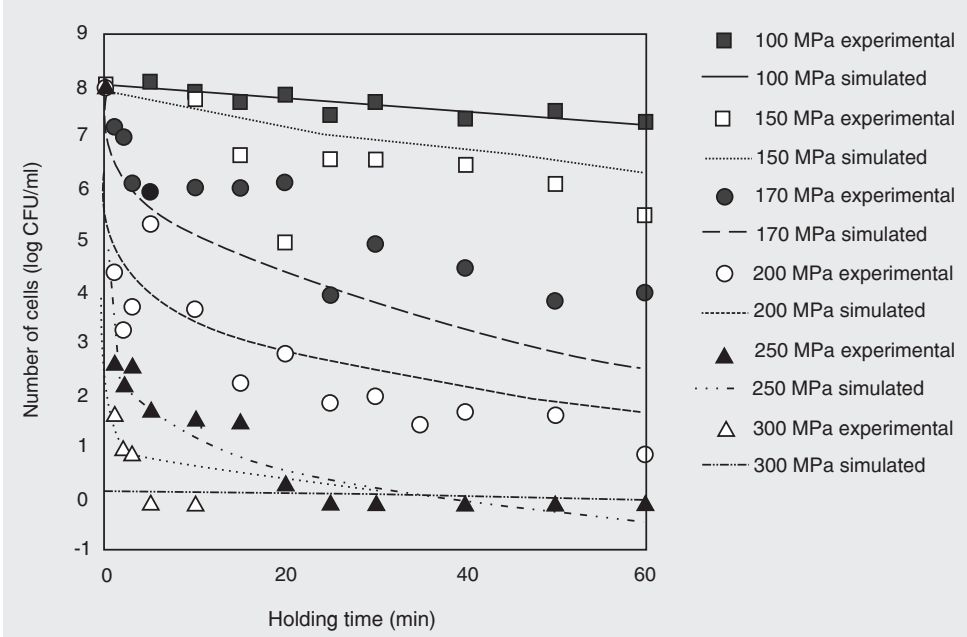

Figure 3. Simulation of pressure inactivation curves of Escherichia coli using the interpolated (recalculated) parameters $b$ and $n$ of Weibull function. where $n_{100 \mathrm{MPa}}$ and $n_{300 \mathrm{MPa}}$ are the values of $n$ for 100 and $300 \mathrm{MPa}$, respectively, and $\alpha_{n}$, $\beta_{n}, \chi_{n}, \delta_{n}, \varepsilon_{n}$, and $\phi_{n}$ are fitting parameters.

As a result, these interpolated parameters made it possible to simulate inactivation curves under any pressure between 100 and $300 \mathrm{MPa}$. By using two predicted equations for $b$ and $n$, each inactivation curve under each holding pressure was simulated and compared with the experimental data set (Figure 3). Although the simulated curve for the $170-\mathrm{MPa}$ treatment predicted a faster inactivation than the experimental curve, the simulated curves for treatments over 200 MPa described the data sets relatively well.

\section{Discussion}

Predictive microbiology for high-pressure inactivation of microorganisms has not been sufficiently investigated compared to heat sterilization. In the present study, a series of experiments were carried out using a model microorganism (E. coli, ATCC $25922)$ in a specific condition $\left(25^{\circ} \mathrm{C}, \mathrm{pH} 6.8\right.$, physiological saline), and the data sets were described by Weibull function. Simulated curves described the experimental data sets and, significantly, the data sets for 250 and $300 \mathrm{MPa}$, where complete inactivation was achieved, were simulated reasonably well in terms of high-pressure food processing, a fact that should not lead to an overestimation of sterilization efficiency.

The Weibull function is often used to describe the thermal inactivation of bacteria (15). It is also good to describe non-thermal processing as well. The Weibull function and its simplified form produces good fits for curves describing the inactivation of a phage by high pressure (16). A pulsed electric field has been used to inactivate foodborne pathogens and the data were described by Weibull function $(17,18)$. Weibull function may be one of the most suitable models for describing not only thermal events but also non-thermal inactivation dynamics of 
microorganisms.

Even though our experimental data were accumulated in a limited range under specific conditions, the prediction method itself can be extended to predict microbial inactivation of other food systems by high pressure at various temperatures, $\mathrm{pH}$, salt con- centration and so forth. Further data accumulation under various conditions is necessary to develop a database on high-pressure inactivation of microorganisms. Validation of predictive models will also be required for each microorganism in each food systems.

\section{References}

1. Barbosa-Canovas GV, Pothakamury UR, Palou E \& Swanson B (1997). Nonthermal Preservation of Foods. Marcel Dekker Inc., New York.

2. Solomon EB \& Hoover DG (2004). Inactivation of Campylobacter jejuni by high hydrostatic pressure. Letters in Applied Microbiology, 38: 505-509.

3. Metrick C, Hoover DG \& Farkas DF (1989). Effects of high hydrostatic pressure on heat-resistant and heat-sensitive strains of Salmonella. Journal of Food Science, 54: 1547-1564.

4. Patterson MF, Quinn M, Simpson R \& Gilmour A (1995). Sensitivity of vegetative pathogens to high hydrostatic pressure treatment in phosphate buffered saline and foods. Journal of Food Protection, 58: 524-529.

5. Syles MF, Hoover DG \& Farkas DF (1991). Response of Listeria monocytogenes and Vibrio parahaemolyticus to high hydrostatic pressure. Journal of Food Science, 56: 1404-1407.

6. Ariefdjohan MW, Nelson PE, Singh RK, Bhunia AK, Balasubramaniam VM \& Singh N (2004). Efficacy of high hydrostatic pressure treatment in reducing Escherichia coli $\mathrm{O} 157$ and Listeria monocytogenes in alfalfa seeds. Journal of Food Science, 69: 117-120.

7. Ellenberg L \& Hoover DG (1999). Injury and survival of Aeromonas hydophila 7965 and Yersinia enterocolitica 9610 from high hydrostatic pressure. Journal of Food Safety, 19: 263-276.

8. Stewart CM, Jewett FF, Cunne CP \& Hoover DG (1997). Effect of concurrent hydrostatic, acidity and heat on the injury and destruction of Listeria monocytogenes. Journal of Food Safety, 17: 23-36.

9. Ross T \& McMeekin TA (1994). Predictive microbiology. International Journal of Food Microbiology, 23: 241-264.

10. McMeekin TA, Olley JN, Ross T \& Ratkowsky DA (1993). Predictive
Microbiology, Theory and Application. John Wiley \& Sons Inc., New York.

11. Baranyi J \& Tamplin ML (2004). ComBase: A common database on microbial responses to food environments. Journal of Food Protection, 67: 1967-1971.

12. Eastern Regional Research Center, US Department of Agriculture Agricultural Research Service, ComBase. [http://wyndmoor.arserrc. gov/combase/]. Accessed December 5, 2004.

13. Eastern Regional Research Center, US Department of Agriculture Agricultural Research Service, Pathogen Modeling Program. [http:// www.arserrc.gov/mfs/pathogen.htm]. Accessed December 5, 2004.

14. Principles and Guidelines for the Conduct of Microbiological Risk Assessment: Codex Alimentarius - Food Hygiene - Basic Texts 2nd edn. [http://www.fao.org/DOCREP/005/Y1579E/y1579e05. htm].

15. van Boekel MAJS (2002). On the use of Weibull model to describe thermal inactivation of microbial vegetative cells. International Journal of Food Microbiology, 74: 139-159.

16. Chen H, Joerger RD, Kingsley DH \& Hoover DG (2004). Pressure inactivation kinetics of phage $\lambda \mathrm{cl} 857$. Journal of Food Protection, 67: 505-511.

17. Álvarez I, Pagán R, Condón S \& Raso J (2002). The influence of process parameters for the inactivation of Listeria monocytogenes by pulsed electric fields. International Journal of Food Microbiology, 87: 87-95.

18. Álvarez I, Raso J, Sala FJ \& Condón S (2003). Inactivation of Yersinia enterocolitica by pulsed electric fields. Food Microbiology, 20: 691-700. 